\title{
Plicae Palmatae on MR in Uterus Didelphys: the Incidence and Morphological Characteristics
}

\author{
Wang Xiaoying \\ Department of Radiology, Peking University First Hospital, Beijing 100034, China.
}

Corresponding Author: Wang Xiaoying, Department of Radiology, Peking University, Beijing 100034, China.

Received date: May 31, 2021: Accepted date: August 10 2021: Published date: October 09, 2021

Citation: Wang Xiaoying (2021) Plicae Palmatae on MR in Uterus Didelphys: the Incidence and Morphological Characteristics J. Clin Case Rep and Stu 2(6); DOI: 10.31579/2690-8808/080

Copyright: () 2021 Wang Xiaoying. This is an open access article distributed under the Creative Commons Attribution License, which permits unrestricted use, distribution, and reproduction in any medium, provided the original work is properly cited.

\begin{abstract}
Objective: To investigate the incidence of plicae palmatae in uterus didelphys and its morphological characteristics on MR imaging.

Methods: We retrospectively collected 37 consecutive female pelvic MR images diagnosed with uterus didelphys between August 2012 and November 2020. Patients with the following conditions were excluded: (a) repeated examination; (b) poor image quality; (c) cervical disease. Axial and coronal T2-weight images and axial three-dimensional (3D) volumetric isotropic T2-weighted acquisition (VISTA) were used to evaluate the ridge of plicae palmatae (RPP). A multiplanar reformation of the cervical axis from 3D-VISTA sequence was performed to measure the height and width of RPP. Nonnormal variables based on the Kruskal-Wallis $\mathrm{H}$ test was used for statistical analysis. A two-tailed test where $\mathrm{P}<0.05$ was considered statistically significant.

Results: Twenty-six cases were finally included in the statistics. The average age was $25.7 \pm 9.0$ years (range, 10-45 years). RPP was observed on both cervices in 16 patients $(61.5 \%)$, only on the left cervix in 3 patients $(11.5 \%)$, and only on the right cervix in 4 patients (15.4\%). There were 3 cases with no RPP observed in any of their cervix (11.5\%).All RPP appear symmetrically on the anterior and posterior walls of the cervix. There was no statistically significant difference in height, width, and height/width of the RPP in the left and right cervix ( $p>0.05)$.
\end{abstract}

Conclusions: RPP is encountered in $88.5 \%$ patients with duplicated uterine cervices in our cohort. This incidence is similar to that reported in women with normal uterus of reproductive age.

Keywords: plicae palmatae, uterine cervix, uterus didelphys, magnetic resonance imaging, incidence

\section{Abbreviations}

3D $=$ three-dimensional

VISTA = volumetric isotropic T2-weighted acquisition

$\mathbf{R P P}=$ the ridge of plicae palmatae

$\mathbf{R A}=$ the anterior wall of the right cervix

$\mathbf{R P}=$ the posterior wall of the right cervix

$\mathbf{L A}=$ the anterior wall of the left cervix

$\mathbf{L P}=$ the posterior wall of the left cervix

SW = Shapiro-Wilk

ADC = apparent diffusion coefficient

DWI = diffusion-weighted imaging

\section{Introduction}

High resolution MRI helps to visualize the zonal anatomy of the uterus. On T2WI MR images, the cervix shows a distinct four-layer structure [1]. The bright intensity in the center represents cervical mucus and the lower intensity adjacent to it represents cervical epithelium. The inner cervical fibrous stroma shows low signal intensity, and the outer loose connective tissue exhibits slightly increased intensity.

Plicae palmatae are cervical folds on the anterior and/or posterior wall, consisting of a median longitudinal ridge and many folds diverging laterally and upward (Figure 1) [2]. The ridges of plicae palmatae (RPP) at the midline are more distinct than the other oblique folds and easier to be observed on MR imaging. The median longitudinal ridges show low intensity on T2WI images, with a frequency of $27-44.5 \%$ on MR imaging in the normal uterus [3-5]. 
Fig.1 Plicae palmatae of cervix. Plicae palmatae are cervical folds on the anterior and/or posterior wall, consisting of a median longitudinal ridge and many folds diverging laterally and upward.

The sign of low intensity at cervical canals could mimic an anomalous uterine septum, so RPP is also called pseudo spectrum [5]. Which indicates their clinical significance. The uterine septum extends from the fundus and extends to the cervix, whereas RPP is limited to the cervical canals. Also, a neonatal case of RPP with a tumorous appearance has been reported [6]. Therefore, it's important for radiologists to accurately distinguish the RPP from the uterine septum or neoplastic lesion to avoid unnecessary hysteroscopy and other invasive procedures.

Currently, there are a few studies on RPP in normal uterus and even less on uterus didephys [7]. This study aims to investigate the incidence of plicae palmatae in uterus didelphys and its morphological characteristics on MR imaging.

\section{Materials and methods}

\section{Study sample}

We retrospectively collected 36 consecutive female pelvic MR images diagnosed with uterus didelphys between August 2012 and November 2020. Patients with the following conditions were excluded from the study: (a) repeated examination of the same patient $(n=3)$; (b) poor image quality $(\mathrm{n}=1)$; (c) cervical disease $(\mathrm{n}=6,2$ cases of cervical hematoma, 1 case of cervical dysplasia, 1 case of endometrial polyp, 2 cases of Nabothian cysts). Figure 2 illustrates the patients' enrollment.

Fig. 2 Diagram shows the patients' enrollment flow.

\section{MR data acquisition}

Patients were scanned on 3.0-Tesla systems (Achieva; Philips Healthcare, Best, The Netherlands) using body phased-array coils for signal reception. The protocol in this study included: (1) Axial and coronal T2-weighted fast spin-echo images (TR/TE, 3000-33760/90-100 msec; ST, 5 mm; intersection gap, $1 \mathrm{~mm}$; FOV, $24-30 \mathrm{~cm} ; 512 \times 512$ matrix), with or without fat saturation; (2) Axial three-dimensional (3D) volumetric isotropic T2-weighted acquisition (VISTA) (TR/TE, 1500/150 ms; ST, $0.8 \mathrm{~mm}$ with no intersection gap; FOV, $18 \mathrm{~cm}$, matrix $180 \times 147$ ).

\section{Methods of observation and measurement of the RPP}

All of the images were analyzed on PACS workstations. The observation and measurement were conducted by one radiologist (Z.S. with more than 3 years of expertise) in consensus with a senior radiologist (X.W., with more than 30 years of experience in genitourinary interpretation). RPP was defined as linear low-intensity structures at the midline of the cervix on MR imaging. The tumorous appearance of RPP is defined as a width greater than or equal to height. To verify the angulated cervix was well observed, axial T2-weighted images, coronal T2-weight images were comprehensively observed regarding the existence of the RPP in each cervix. In case of doubt, reconstruction of the cervical axis from the axial 3D-VISTA was used to observe when necessary. A reconstructed maximum cross-section of the RPP from the axial 3D-VISTA was also performed to measure the height and width. Four sites were measured respectively: the anterior and posterior wall of the right cervix (RA and $\mathrm{RP}$ ) and the anterior and posterior wall of the left cervix (LA and LP), as shown in Figure 3.

Fig.3 a) and b) Axial 3D-VISTA image and coronal T2-weighted images in a 30-year-old woman with uterus didelphys. Longitudinal ridges show distinct linear low signal intensity on the anterior and posterior walls of both uterine cervix (arrows). c) An example of measurement method for the ridge of plicae palmatae (RPP) on the anterior wall of the left cervix. A reconstructed maximum cross-section of the RPP from the axial 3DVISTA was performed to measure the height and width. RA: the anterior wall of the right cervix; RP: the posterior wall of the right cervix; LA: the anterior wall of the left cervix; LP: the posterior wall of the left cervix.

\section{Statistical analysis}

A Shapiro-Wilk (S-W) test was conducted to test the normality of all measurements. Continuous variables were expressed as mean \pm standard deviation or median (interquartile range). Categorical variables were summarized as counts (percentage). Non-normal variables based on the Kruskal-Wallis $\mathrm{H}$ test was used to compare the measurements of the RPP in the left and right cervix. Statistical analysis was performed with programming language (python version 3.5.0; Python Software Foundation; https://www.python.org) and IBM SPSS Statistics version 24.0. A two-tailed test where $\mathrm{P}<0.05$ was considered statistically significant.

\section{Results}

\section{The incidence of the RPP on MR}

Twenty-six cases were finally included in our study. The average age of the 26 patients was $25.7 \pm 9.0$ years (range, 10-45 years). RPP is encountered in $88.4 \%$ patients with duplicated uterine cervices in our cohort. The number of RPP observed on both cervices, left cervix only, and right cervix only were $16(61.5 \%), 3(11.5 \%)$, and $4(15.4 \%)$, respectively. There were $3(11.5 \%)$ cases with no RPP observed in any of their cervix.

\section{The morphological characteristics of the RPP}

All RPP appeared symmetrically on the anterior and posterior walls of the cervix. In 23 individuals with RPP visible in unilateral or bilateral cervices, the mean height, width, and height/width of RA, RP, LA, and LP were summarized in table 1, Figure 4 . There isn't a statistically significant difference of the measurements between the left and right cervix ( $p>0.05$ ). In all the 26 uterus didelphys included in the study, only one case of RPP showed tumor-like structure (Figure 5), the rest were line-like structures with height greater than width (Figure 3).

a), b) and c) are Box plots indicating the height, width, and height/width of the ridge of plicae palmatae in four locations of the cervical canal, respectively. RA: the anterior wall of the right cervix; RP: the posterior wall of the right cervix; LA: the anterior wall of the left cervix; LP: the posterior wall of the left cervix. RPP: the ridge of plicae palmatae. The whisker lengths are limited to a maximum of 1.5 times the interquartile range. Each box represents the $95 \%$ confidence interval near the median.

The ridge of plicae palmatae (RPP) showed tumor-like structure in a 28year-old woman. a) and b) Axial 3D-VISTA image and coronal T2weighted fat saturated image. The RPP at the anterior wall of the left cervix shows a tumorous appearance (arrow) with height, width of $8.3 \mathrm{~mm}$ and $8.5 \mathrm{~mm}$. The width of RPP is greater than the height but it is still low signal density; c) and d) Axial contrast-enhanced T1-weighted fat saturated images during the equilibrium phase $(2 \min 30 \mathrm{~s}$ after the injection) and delayed phase (10 min after the injection) show the presence of iso-enhancement (arrow); e) and f) No restricted diffusion (arrow) is observed on diffusion-weighted imaging (high $b$ value of 1000 $\mathrm{s} / \mathrm{mm}^{2}$ ) and apparent diffusion coefficient (ADC) maps. RA: the anterior wall of the right cervix; RP: the posterior wall of the right cervix; LA: the anterior wall of the left cervix; LP: the posterior wall of the left cervix. RPP: the ridge of plicae palmatae.

The mean height, width, and height/width of the ridge of plicae palmatae $\mathrm{NA}=$ not available, RA: the anterior wall of the right cervix; RP: the posterior wall of the right cervix; LA: the anterior wall of the left cervix; LP: the posterior wall of the left cervix. Data are presented as the median (interquartile range) and mean \pm standard deviation. * $\mathrm{P}$ values for nonnormal variables are based on the Kruskal-Wallis $\mathrm{H}$ test. 


\section{Discussion}

In this retrospective study, we searched MR studies of female patients in our institution and detected 23 RPP in 26 cases with uterus didelphys. Embryologically, bilateral Mullerian tubes fuse to form a normal uterus. Failure of the intervening septum regression can result in anomalous uterine septum or other Mullerian duct anomalies. Uterus didelphys is a typical situation of Mullerian anomalous. Due to the RPP are located at the midline of the cervical canal, they were once hypothesized to be a remnant of a developmental Mullerian ductal fusion. Recently, Takahata et al.[7] Reported on a case of uterus didelphys observed with RPP, the observation of the RPP on the duplicated uterine cervices indicates the plicae palmatae is an inherent structure of the cervical canal, not a remnant of fused Mullerian duct. This report refuted the previous hypothesis. The goal of this study was to observe the relationship between RPP and Mullerian anomalous. Based on our observations, we favor the theory of the RPP was not a remnant of Mullerian ductal fusion, and we found they can be observed in either one or both cervix.

Previous studies have found that RPP is more frequently visualized in normal uterus of reproductive age, with a rate of $58.4 \%$ for those under 45 years of age [8]. The study also found that women in their 30 s had the highest frequency $(71.4 \%)$ of visualization of RPP on MR. Our study summarized a frequency of $88.5 \%$ in RPP of uterus didelphys on MR, which was higher than that of a normal uterus but was close to the group of 30s. This may be due to the fact that the age of our participants was concentrated around child-bearing age (25.7 \pm 9.0 years). Previous studies on RPP have been conducted on axial and/or coronal T2-weighted images. To date, measurements of the RPP are absent. We used axial 3D T2WI to observe more detail and measure the height and width of the RPP by using multi-planar reformation to provide anatomical data on MR images.

In our study cohort, only one RPP that mimics a tumor has been observed. The width of RPP is greater than the height but it is still low signal density and with no restricted diffusion on diffusion-weighted imaging (DWI). A neonatal case of tumorous RPP has been reported and described the sonographic and DWI findings [6]. We believe that the width of the RPP can appear greater than the height under the premise that the signal characteristics are consistent with the cervical folds.

One distinct limitation of our study is that our diagnosis was not identified histologically. The findings that restricted to the cervix without extending to the uterine fundus, low T2WI signal density, and does not traverse the entire cervix could be confirmed that the structure is RPP. Another limitation is the measurement error due to the small size of the RPP. We adopted the measurement plane jointly confirmed by two radiologists in consensus and tried to magnify the image during the measurement to reduce the measurement error. A third limitation is that the number of patients included in our study is small and the mean age is mostly around child-bearing age, which limits age-stratified studies based on hormonal state. The fourth limitation is lack of RPP frequency comparison between uterus didelphys and normal uteri over the same period, this may be another study in the future.

\section{Conclusion}

RPP is frequently encountered in duplicated uterine cervices, and most of them appear symmetrically on the anterior and posterior walls without any difference in height, width, and height/width from one another. It seems that RPP might be an inherent structure of the uterine cervical canal, instead of a remnant of fused Mullerian ducts.

This research did not receive any specific grant from funding agencies in the public, commercial, or not-for-profit sectors [2].

\section{Reference}

1. L.M. Scoutt, T.R. McCauley, S.D. Flynn, D.J. Luthringer, S.M. McCarthy (1993) Zonal anatomy of the cervix: correlation of MR imaging and histologic examination of hysterectomy specimens, Radiology, 186(1), 159-62.

2. R.W. Kistner, (1980) Gynecology Principles and Practice. Chicago, IL: Year Book Medical Publishers.

3. A. Takahata, T. Koyama, K. Yamada, T. Nishimura, S. Fujii, K. Togashi, (2008) Plicae palmatae of the cervical canal visualized on MRI, The British journal of radiology 81(961), e4-6.

4. A. Takahata, T. Koyama, A. Kido, M. Kataoka, S. Umeoka, et al. (2009) The frequency of the plicae palmatae in the uterine cervix on MR imaging, Abdominal imaging 34(2), 277-9.

5. A.K. El Jack, E.S. Siegelman, (2007) "Pseudoseptum" of the uterine cervix on MRI, Journal of magnetic resonance imaging : JMRI 26(4), 963-5.

6. M. Kitami, (2001) Plicae palmatae in neonates: tumor mimic on sonography, Journal of medical ultrasonics, 45(1), 193-196.

7. A. Takahata, T. Koyama, (2012) Plicae palmatae of the cervical canals in uterus didelphys: MR imaging, Abdominal imaging 37(5), 912-3.

8. F. Uysal, S. Cevizci, M. Reşorlu, M. Gencer, A. Uysal, (2015) Plicae palmatae: an anatomical uterine cervix variation to be considered on magnetic resonance images, Clinical imaging 39(2), 270-2. 